\title{
Resúmenes del Premio José Félix Patiño Restrepo
}

\author{
https://doi.org/10.35454/rncm.v2n1.065
}

PROTOCOLOS

\begin{abstract}
PARÁMETROS DE COMPOSICIÓN CORPORAL E INMUNOLÓGICOS DE PACIENTES CON VIH. HOSPITAL NACIONAL GUILLERMO ALMENARA IRIGOYEN- 2017 Y 2018
\end{abstract}

Bettyna Benavente Grández

Introducción: siendo la bioimpedancia una herramienta no invasiva y de bajo costo para la evaluación de composición corporal, se han desarrollado estudios que exploran estos parámetros en pacientes con VIH, en Brasil se encontró como promedio de ángulo de fase $5.2^{\circ}$ para pacientes con $\mathrm{VIH}$, en México se concluyó que un punto de corte de $5.15^{\circ}$ de ángulo de fase es un indicador de desnutrición, así mismo, en Venezuela se ha descrito correlación significativa entre masa grasa y tejido adiposo abdominal respecto a Linfocitos CD4.

Objetivos: el objetivo es valorar parámetros de composición corporal e inmunológicos, así como la relación entre ambos, en pacientes con VIH que reciben TARGA en el Hospital Nacional Guillermo Almenara, evaluados con bioimpedancia durante los años 2017 y 2018.
Métodos: estudio descriptivo, correlacional, retrospectivo y transversal. Se tomarán lo datos de la Ficha Nutricional de Bioimpedancia: género, edad, peso, talla, datos obtenidos mediante Bioimpedancia: Ángulo de Fase, Masa libre de grasa, Masa grasa y tejido adiposo abdominal, se registrará la carga viral según LCD4 de la historia clínica del paciente. Se analizarán los datos de la población integrada por 130 fichas.

Perspectiva y resultados esperados: se espera poder determinar el promedio de ángulo de fase, así como corroborar la correlación entre masa grasa, tejido adiposo abdominal respecto a linfocitos CD4.

Palabras clave: VIH, bioimpedancia, composición corporal, ángulo de fase.

Hospital Nacional Guillermo Almenara, Lima, Perú. bettynagrandez@hotmail.com

\section{EFECTO DE UN PROGRAMA DE INTERVENCIÓN NUTRICIONAL COMBINADA CON ENTRENAMIENTO FÍSICO SOBRE LA DISFUNCIÓN MUSCULAR DE PACIENTES CON EPOC}

María Camila Pineda Zuluaga

Introduccion: la Enfermedad Pulmonar Obstructiva Crónica (EPOC), es la cuarta causa de muerte en todo el mundo, pero se prevé que será la tercera en el 2020. Esta condición se asocia con efectos sistémicos extra pulmonares como la disfunción muscular que tiene repercusiones negativas sobre la capacidad funcional y la calidad de vida relacionada con la salud. La European Respiratory Society (ERS) sugiere incluir en el tratamiento de estos pacientes, intervenciones que consistan en complementos nutricionales combinados con entrenamiento físico que eviten principalmente la pérdida de peso y la disfunción muscular.

Objetivo: evaluar los efectos de un programa de intervención nutricional combinado con entrenamiento físico, con la hipótesis de que los pacientes beneficiarios de la intervención combinada tendrán una mejor función muscular al finalizar la intervención.
Métodos: a todos los participantes del estudio se les evaluarán los biomarcadores (fibrinógeno, IL-6 y 8, TNF- a, bilirrubina total, PCR sistémica y leucocitos), variables de función pulmonar (volumen espiratorio forzado en el primer segundo $\left(\mathrm{FEV}_{1}\right.$ ), la capacidad vital forzada (CVF) y el cociente $\mathrm{FEV}_{1}$ / CVF), composición corporal (antropometría y bioimpedancia) y capacidad funcional (dinamometría manual, SPPB y 6MWT) antes y después de la intervención nutricional combinada con entrenamiento físico, que consistirá en la administración de un complemento nutricional diario combinado con 3 sesiones de entrenamiento físico semanal, durante 12 semanas.

Resultados esperados: se espera lograr efectos biológica y clínicamente favorables sobre la disfunción muscular de pacientes con EPOC, tras una intervención nutricional combinada con entrenamiento físico.

Palabras clave: EPOC, intervención, nutrición, entrenamiento físico.

Universidad de Caldas, Manizales, Colombia.

\section{ASOCIACIÓN ENTRE NIVELES DE ZINC Y TRASTORNO DEPRESIVO MAYOR, MANIZALES PERÍODO 2018-2019: ESTUDIO ZINDEPA}

\section{Laura Victoria Sánchez Díaz}

Introducción: hay un interés creciente por demostrar la relación entre la patología mental y el déficit de micronutrientes, para tener una alternativa en el abordaje terapéutico estos pacientes. La evidencia recolectada en varios estudios ha revelado una conexión entre niveles bajos de zinc en plasma y depresión. En un estudio con ancianos hospitalizados, se encontró que el $28 \%$ de la población estudiada presentaba concentraciones de zinc inferiores a $10.7 \mu \mathrm{mol} / \mathrm{L}$.
Objetivo: describir la asociación entre los niveles de zinc sérico y el trastorno depresivo mayor en adultos hospitalizados en la clínica psiquiátrica San Juan de Dios en la ciudad de Manizales-Caldas, con la hipótesis de que los niveles de zinc sérico están disminuidos en estos pacientes.

Métodos: para esta investigación se utilizarán 2 instrumentos, el cuestionario de depresión de Beck y el recordatorio alimentario de 24 horas. Se 
realizarán visitas al servicio de hospitalización de la Clínica psiquiátrica San Juan de Dios en Manizales, en las cuales a los pacientes con diagnóstico de depresión que cumplan los criterios de selección, serán informados sobre la posibilidad de participar en el estudio. En caso de aceptar, el investigador realizará el procedimiento del consentimiento informado. Se procederá al diligenciamiento de los cuestionarios mencionados y se les extraerá una muestra de sangre.
Resultados esperados: se espera encontrar que los niveles de zinc sérico están disminuidos en los pacientes con trastorno depresivo en estos pacientes.

Conclusiones esperadas: esto sugeriría que la suplementación con zinc podría ser una herramienta coadyuvante para la terapia integral de estos pacientes.

Palabras clave: zinc, trastorno depresivo mayor, Manizales.

Universidad de Caldas, Manizales, Colombia.

\section{COMPOSICIÓN CORPORAL EN PACIENTES CON NEOPLASIAS MALIGNAS INCIDENTES: SEGUIMIENTO A 6 MESES EN CENTRO ONCOLÓGICO EN LA CIUDAD DE MANIZALES}

Juan Daniel Duque

Introducción: la medición de la composición corporal se ha vuelto relevante en las últimas dos décadas en los pacientes con cáncer. En estos pacientes la investigación relacionada con la composición corporal es escasa. Es a partir de 2009 con el estudio del Dr. Crawford que se empezaron a describir asociaciones entre los parámetros de composición corporal y la sobrevida.

Objetivo: establecer los cambios en la composición corporal y su efecto sobre la funcionalidad y desenlaces clínicos a los 3 y 6 meses en pacientes con diagnóstico de cáncer de pulmón, mama, estómago, próstata, colon, recto, páncreas y linfoma que consultan a un centro oncológico en la ciudad de Manizales, Caldas.

Métodos: los datos serán recolectados a través de una encuesta a los pacientes referidos por Oncólogos de Occidente durante junio de 2019 y junio de 2020 que cumplan los criterios de inclusión.
Resultados esperados: se espera encontrar que la composición corporal de los pacientes con diagnóstico de cáncer de pulmón, mama, estómago, próstata, colon, recto, páncreas y linfoma, evaluada a partir del análisis de bioimpedancia eléctrica, se correlaciona con la funcionalidad y los desenlaces clínicos a los 3 y 6 meses de seguimiento.

Conclusiones esperadas: lo anterior dará a conocer nuevas herramientas diagnósticas, que resultan útiles para la evaluación integral del paciente oncológico y de esta manera poder hacer un soporte nutricional temprano para evitar los efectos adversos sobre la composición corporal.

Palabras clave: composición corporal, neoplasias malignas, seguimiento.

Universidad de Caldas. Manizales, Colombia.

\section{RELACIÓN DE SARCOPENIA, RIESGO CARDIOMETABÓLICO, MARCADORES DE INFLAMACIÓN Y VALORES DE VITAMINA D EN MUJERES PERIMENOPAUSICAS Y MENOPAÚSICAS}

Ludwig R. Álvarez Córdova, Diana Fonseca Pérez, Domenique Chavez Pérez, Gabriela Gómez Vaca, Cecilia Arteaga Pazmiño

Introducción: la sarcopenia está catalogada como un desorden progresivo y generalizado del musculo estriado asociado a un riesgo mayor de presentar caídas, fracturas, discapacidad física y muerte. La prevalencia de la misma aumenta con el aumento de las décadas de vida y cambios de la composición corporal.

Objetivo: determinar la prevalencia de sarcopenia, riesgo cardiometabólico, marcadores de inflamación y niveles de vitamina $D$ en la población determinada.

Hipótesis: la sarcopenia en mujeres perimenopaúsicas y menopaúsicas estará relacionado a la presencia de riesgo cardiometabólico, valores elevados de marcadores de inflamación sistémicos y niveles séricos bajos de vitamina $D$.

Métodos: estudio descriptivo, observacional transversal de diagnóstico y estadificación. Se utilizarán los criterios del EWGSOP2 valorando la fuerza prensil de mano con un dinamómetro, la masa múscular apendicular utililzando bioimpedancia, la funcionabilidad muscular utilizando el Short Battery Test.
Perspectivas: se relacionará las variables de diagnóstico de sarcopenia en mujeres perimenopaúsicas y menopaúsicas con la presencia de riesgo cardiometabólico, marcadores de inflamación sistémicos y bajos niveles séricos de vitamina D. La estadísticas serán determinadas por medio de pruebas de Chi Cuadrado y valores de $\mathrm{p}$ - value para verificar la relación directa de la hipótesis declarada, cuando dichos valores sean inferiores a 0.05 con análisis de correlacion.

Resultados esperados: investigar tempranamente la sarcopenia en las mujeres perimenopaúsicas y menopaúsicas según el algoritmo propuesto por el EWGSOP2 y relacionarlo con la presencia de riesgo cardiometabólico, presencia de marcadores de inflamación y bajos niveles de vitamina D.

Palabras clave: sarcopenia, bioimpedancia, fuerza prensil de mano, marcadores de inflamación.

Universidad Católica de Santiago de Guayaquil, Ecuador.

\section{EFICACIA DE LACTOBACILLUS REUTERI Y GLUTAMINA EN EL CONTROL DE LA DIARREA EN PACIENTES CRÍTICOS}

María Elena Goiburu, Rossana Salinas, Larissa Miranda

Introducción: aunque la diarrea es una complicación común en pacientes críticos, el impacto sobre la recuperación total y el estado nutricional está subestimado. Los escasos estudios del efecto de probióticos y glutamina en pacientes con diarrea, hace necesario evaluar estrategias de soporte nutricional.
Objetivo: determinar la eficacia de un suplemento de Lactobacillus Reuteri comparado con otro de Lactobacillus Reuteri+ glutamina y un tercer grupo placebo, en el control de la diarrea considerando duración y frecuencia de episodios en pacientes críticos. 
Hipótesis: Ho: No hay diferencia de duración de diarrea con probióticos y probióticos + glutamina. Ho: No hay diferencia de frecuencia de diarrea con probióticos y probióticos + glutamina. HA: Hay menor duración de diarrea con probióticos + glutamina. HA: Hay menor frecuencia de diarrea con probióticos + glutamina.

Métodos: estudio experimental doble ciego aleatorizado de pacientes con diarrea en Terapia Intensiva Adultos del Hospital de Clínicas-Paraguay. Se aleatorizará a los pacientes en tres grupos de intervención, se suministrará: grupo A: suplemento con probiótico (BioGaia ${ }^{\oplus}$ ) diluido en $100 \mathrm{ml}$ de agua; grupo B: suplemento con glutamina + probiótico (GlutaPak $\mathrm{R}^{\odot}$ ) diluido en $100 \mathrm{ml}$ agua; grupo C: placebo $100 \mathrm{ml}$ de agua.

Perspectivas y resultados esperados: se espera que el uso de suplementos con probióticos asociados a glutamina en pacientes críticos con diarrea, disminuya la frecuencia y duración de los episodios, con lo cual poder elaborar según los resultados obtenidos, protocolos específicos y claros sobre el beneficio de este tipo de suplementos que contribuyan para dar solución a dicha situación de los pacientes.

Palabras clave: lactobacillus reuteri, glutamina, paciente crítico, diarrea.

Hospital de Clínicas. Universidad Nacional de Asunción. Asunción, Paraguay.

\title{
EFECTO DEL CONSUMO DE UNA FÓRMULA ENTERAL HIPERPROTEICA SOBRE LA COMPOSICIÓN CORPORAL Y EL FACTOR DE CRECIMIENTO ENDOTELIAL VASCULAR EN PACIENTES CON CÁNCER HEMATOLÓGICO
}

Mónica Patricia Bejarano, Vanessa Fuchs Tarlovsky, Karolina Álvarez Altamirano, Christian Omar Ramos Peñafiel, Gilberto Barranco Lampón, María Dolores Arias Soberon

Introducción: los pacientes con cáncer hematológico tienen alto riesgo de desarrollar sarcopenia, la cual disminuye la respuesta al tratamiento, la capacidad funciona y la calidad de vida. Los medicamentos usados para el tratamiento de esta patología son inhibidores de angiogénesis que afectan procesos fisiológicos importantes de adipogénesis y anabolismo muscular. El factor de crecimiento endotelial vascular (VEGF) y sus receptores, juegan un rol crucial en ambos, la angiogénesis normal y maligna. La activación de la vía del VEGF lleva a la activación celular endotelial, proliferación y sobrevivencia. El uso de inhibidores de la angiogénesis en pacientes con cáncer pone en duda su efecto sobre la composición corporal y el peso y es una terapia novel utilizada en estos pacientes.
El objetivo de este estudio es comparar el efecto del uso de una fórmula enteral hiperproteica e hiperenergética contra una fórmula enteral estándar que es el tratamiento estándar sobre la tolerancia a la misma, composición corporal, días de estancia hospitalaria, calidad de vida, fuerza muscular asociado con los niveles plasmáticos del VEGF en pacientes con cáncer hematológico durante la quimioterapia.

Se diseñó un ensayo clínico abierto, en el cual se seleccionará una muestra de 37 pacientes por grupo a quienes se les administrará una parte sus requerimientos nutricionales cubiertos con una fórmula polimérica hiperproteica versus fórmula estándar durante los 21 días que dura el primer ciclo de tratamiento antineoplásico.

Palabras clave: fórmula enteral hiperproteica, composición corporal, fuerza muscular, VEGF, cáncer hematológico.

\section{COMPOSICIÓN DE BIOCOMPONENTES EN LECHE HUMANA POSTERIOR A PASTEURIZACIÓN HOLDER EN EL BANCO DE LECHE DEL HOSPITAL GENERAL DE MEDELLÍN}

\author{
Nelly Patricia Castillejo Padilla1,2, Daniela Vanegas Otálvaro', Natalia Zuluaga Arroyave', Alejandra Agudelo Martínez¹,2, Natalia Lopera Sánchez.
}

Introducción: la leche humana constituye la principal alternativa de alimentación para neonatos y lactantes por su aporte nutricional e inmunocomponentes. Existen situaciones donde es inevitable la separación del binomio madre-hijo o hay contraindicaciones de la lactancia materna, poniendo en riesgo la salud a corto y largo plazo del lactante. Debido a lo anterior, surge la estrategia de bancos de leche a nivel mundial para contribuir al consumo de leche materna, incluso en condiciones de separación del binomio, donde se realiza la pasteurización de leche bajo metodologías que implican diferentes etapas desde la recolección hasta la distribución, que podrían afectar el contenido y calidad de nutrientes y compuestos inmunogénicos.

Objetivo: determinar el contenido de Inmunoglobulina A Secretora y Lactoferrina en leche humana donada, en cada etapa que implica el proceso

Universidad CES, Docentes-investigadoras Facultad de Ciencias de la Nutrición y los Alimentos. Medellín, Antioquia.

2 Profesional banco de leche humana, Hospital General de Medellín, Colombia. de pasteurización y distribución al neonato receptor del Banco de leche del Hospital General de Medellín.

Métodos: se realizará un estudio longitudinal donde se evaluará mediante la técnica ELISA el contenido en inmunoglobulina A secretora y Lactoferrina en 4 etapas requeridas para la pasteurización por el método Holder de leche humana.

Hipótesis: la leche humana sometida a las etapas que implica la pasteurización y distribución, presenta disminución en el contenido de Inmunoglobulina A secretora y Lactoferrina.

Resultados esperados: el presente estudio ampliará la información disponible sobre la composición de leche humana pasteurizada y constituirá el inicio para el planteamiento de estudios que evalúen la biodisponibilidad de biocomponentes.

Palabras clave: pasteurización Holder, leche humana pasteurizada, banco de leche humana.

\section{ASOCIACIÓN DE LA FUERZA DE AGARRE CON EL NIVEL DE ACTIVIDAD FÍSICA EN DOCENTES UNIVERSITARIOS EN EL 2019}

María Alejandra Alfonso Barón, Oscar Fernando Fonseca Vargas, María Paula García Zamora, María Alejandra Ortiz Ávila, Alejandro Pinzón Mahecha, Martha Andrea Rodríguez Castelblanco, Gustavo A. Díaz

Introducción: la ocupación está relacionada con el nivel de actividad física y la fuerza de agarre en adultos. Se han encontrado reportes sobre fuerza de agarre o actividad física en docentes, pero no se dispone de información sobre la asociación de ambos eventos en docentes universitarios. 
Objetivo: determinar la asociación entre fuerza de agarre y nivel de actividad física en docentes universitarios durante el segundo semestre del 2019.

Métodos: estudio transversal y analítico en 184 docentes universitarios seleccionados aleatoriamente en la universidad a partir de una lista. Se medirán variables demográficas, académicas y antropométricas. A nivel laboral se detallaran aspectos de intensidad y labores realizadas mediante un cuestionario estructurado diseñado para la investigación. La actividad física se medirá mediante podómetro y el cuestionario IPAQ-SF. La fuerza de agarre se medirá con el dinamómetro Jamar. La asociación será evaluada mediante un modelo lineal univariado y multivariado.

Resultados esperados: A nivel descriptivo, se espera detallar la prevalencia de actividad física y promedio de fuerza de agarre en los docentes una universidad en Bogotá. En cuanto a lo analítico, se prevé establecer la asociación entre la fuerza de agarre y el nivel de actividad física en docentes universitarios. Los resultados permitirán a la universidad establecer intervenciones que mejoren el desempeño físico y contribuirá al entendimiento del fenómeno estudiado.

Palabras clave: actividad física, fuerza de agarre, docentes universitarios

Universidad El Bosque. Facultad de Medicina.

\section{EFECTO OBESOGÉNICO RELACIONADO CON EL CONSUMO DE EDULCORANTES ACALÓRICOS (ACELSUFAME K) EN FUNCIONARIOS CON SOBREPESO DE LA UNIVERSIDAD MARIANA}

Nelson Enrique Conde Parada

Introducción: el trabajo de investigación presenta la descripción y el análisis del efecto obesogénico relacionado con el consumo de edulcorantes acalóricos (acelsufame K), en funcionarios con diagnóstico de sobrepeso en la Universidad Mariana. Teniendo que a nivel mundial el consumo de este tipo de sustancias se ha incrementado considerablemente durante los últimos 10 años, es necesario identificar la posible asociación con la obesidad siendo esta alteración una situación establecida como un problema actual de salud pública. Por ello como objetivo de este estudio se pretende analizar el efecto ocasionado durante el consumo del edulcorante acelsufame $\mathrm{K}$, y la posible

Universidad Mariana, Colombia. relación o no con el efecto obesogénico. La investigación se realizará teniendo el estudio analítico de casos y controles, en donde se realizarán métodos para la identificación de la ingesta dietética, la composición corporal, el nivel de actividad física, y parámetros bioquímicos como los niveles de leptina y hemoglobina glicosilada en sangre, parámetros que se encuentran estrechamente unidos al efecto y que se determinan con el fin de aportar mayor conocimiento en el uso de los edulcorantes en las diferentes condiciones patológicas o preferenciales de consumo en la población en general.

Palabras clave: obesidad, edulcorantes acalóricos, efecto obesogénico.

\section{VARIABILIDAD DEL ÍNDICE DE LA MASA MUSCULAR COMO PREDICTOR DE MORTALIDAD EN PACIENTES CRÍTICAMENTE ENFERMOS}

Rolando Medina, Juan Sanjuan, Sandra Milena Rojas, Wilmer Botache

Introducción: la sarcopenia consiste en la pérdida del volumen y la función muscular de las personas, dicha variación se encuentra relacionada a diferentes factores como la edad y el compromiso de la reserva fisiológica. De manera creciente en la literatura se ha introducido la relación de la sarcopenia con desenlaces como mortalidad, requerimiento de soporte ventilatorio, complicaciones postoperatorias, entre otras. El abordaje de esta entidad clínica se ha estimado de diferentes maneras entre las cuales se incluyen medidas antropométricas e imaginológicas, siendo la tomografía computarizada ampliamente utilizada, sin embargo, también se han descrito aproximaciones diagnosticas basadas en imágenes como el uso de la ultrasonografía para el cálculo del índice de la masa muscular (IMU), el cual cuenta con protocolos estandarizados para su medición.
Objetivos: Evaluar la relación entre la variabilidad del índice de la masa muscular en miembros inferiores con la mortalidad al egreso en pacientes críticamente enfermos.

Hipótesis: En el marco de la presente investigación se plantea la hipótesis nula en la cual no existe una relación entre la variabilidad del índice de la masa muscular en miembros inferiores con la mortalidad al egreso en pacientes críticamente enfermos admitidos a la unidad de cuidado intensivo (UCI).

Métodos: las mediciones por ecografia de (IMU), se realizaran en dos tiempos diferentes por el mismo evaluador para identificar la variabilidad.

Resultados esperados: rendimiento de las medidas obtenidas del índice de masa muscular mediante ultrasonografía en pacientes admitidos a la UCI. Identificación de la magnitud de la variabilidad del IMU en Mis.

\section{ASOCIACIÓN DE LA FUERZA DE AGARRE CON EL NIVEL DE ACTIVIDAD FÍSICA EN DOCENTES UNIVERSITARIOS EN EL 2019}

María Alejandra Alfonso Barón, Oscar Fernando Fonseca Vargas, María Paula García Zamora, María Alejandra Ortiz Ávila, Alejandro Pinzón Mahecha, Martha Andrea Rodríguez Castelblanco

Introducción: la ocupación está relacionada con el nivel de actividad física y la fuerza de agarre en adultos. Se han encontrado reportes sobre fuerza de agarre o actividad física en docentes, pero no se dispone de información sobre la asociación de ambos eventos en docentes universitarios.

Objetivo: determinar la asociación entre fuerza de agarre y nivel de actividad física en docentes universitarios durante el segundo semestre del 2019.
Métodos: estudio transversal y analítico en 184 docentes universitarios seleccionados aleatoriamente en la universidad a partir de una lista. Se medirán variables demográficas, académicas y antropométricas. A nivel laboral se detallaran aspectos de intensidad y labores realizadas mediante un cuestionario estructurado diseñado para la investigación. La actividad física se medirá mediante podómetro y el cuestionario IPAQ-SF. La fuerza de agarre se medirá 
Rev. Nutr. Clin. Metab. 2019;2(1):89-105.

con el dinamómetro Jamar. La asociación será evaluada mediante un modelo lineal univariado y multivariado.

Resultados esperados: a nivel descriptivo, se espera detallar la prevalencia de actividad física y promedio de fuerza de agarre en los docentes

Universidad El Bosque. Facultad de Medicina, Bogotá, Colombia. una universidad en Bogotá. En cuanto a lo analítico, se prevé establecer la asociación entre la fuerza de agarre y el nivel de actividad física en docentes universitarios. Los resultados permitirán a la universidad establecer intervenciones que mejoren el desempeño físico y contribuirá al entendimiento del fenómeno estudiado.

Palabras clave: actividad física, fuerza de agarre, docentes universitarios. 


\title{
Resúmenes Premio José Félix Patiño Restrepo
}

\author{
TRABAJOS DE GRADO
}

\section{DISEÑO DE PROTOCOLO PARA MEDICIÓN Y REPORTE DE PÉRDIDAS Y DESPERDICIO DE ALIMENTOS GENERADOS EN SERVICIO DE ALIMENTACIÓN HOSPITALARIO}

Maryi Alejandra Orjuela Herrera

Introducción: las pérdidas y desperdicio de alimentos (PDA) generados durante la cadena alimentaria ha sido ampliamente estudiada, así como el impacto que ocasiona a nivel económico, social y ambiental. Sin embargo, en el ámbito nacional en servicios de alimentación (SA) hospitalarios [restauración hospitalaria], no se ha documentado la medición y reporte las misma.

Objetivo: el objetivo del presente trabajo fue diseñar un protocolo para la medición y reporte de PDA en el un SA ubicado en una Institución hospitalaria de cuarto nivel.

Metodología: se realizó un estudio cuantitativo, observacional, durante un mes, con muestra a conveniencia de las PDA generadas en diferentes sectores del S.A y en los servicios de hospitalización; para lo cual se separaron

Pontificia Universidad Javeriana. maryi.orjuela@javeriana.edu.co físicamente, clasificaron y pesaron. Se estandarizaron los procesos para la medición, reporte de PDA y se propuso una escala para evaluar la calidad de la alimentación ofrecida a partir de la generación de éstas. El SA se clasificó como tolerable, con PDA de $11,4 \%$; de los cuales $6,2 \%$ se genera en el sector de producción y 5,2\%, después del servicio.

Resultados: los factores asociados con mayores PDA corresponden a fatiga laboral, experticia, agilidad, responsabilidad del personal manipulador durante el desarrollo de operaciones de producción, y rechazo de los pacientes a algunas preparaciones ofrecidas: principalmente de los componentes de sopa y jugo.

Conclusión: se recomienda realizar futuros estudios para validar la escala propuesta para evaluar la calidad del SA, con base en la generación de PDA.

Palabras clave: cuantificación de pérdida y desperdicio de alimentos, servicio de alimentación, protocolo pérdidas y desperdicio.

\section{ESTADO NUTRICIONAL Y CONDICIÓN FÍSICA DE LOS FUTBOLISTAS DEL CLUB DEPORTIVO TALENTOS GUAJIROS CATEGORÍA PREJUVENIL DEL DISTRITO DE RIOHACHA}

Yaina Panciera di Zoppola, Maria del Rocio Ortiz, Dilver Garcia, Junior Cardona, Oscar Ibarra

Introducción: el estado nutricional y la condición física son factores asociados que se hace necesario investigar a fin de obtener mejores logros deportivos.

Objetivos: la finalidad del proyecto fue conocer el estado nutricional y condición física de los futbolistas de la categoría pre-juvenil del club deportivo Talentos Guajiros del Distrito de Riohacha.

Métodos: estudio descriptivo transversal, se realizó valoración nutricional a través de los indicadores peso/talla, para evaluar la condición física se aplicó una serie de baterías, para la aplicación de los test y mediciones antropométricas se tuvo en cuenta los protocolos internacionales y consideraciones éticas para toma de mediciones.
Resultados: más del $55 \%$ de los adolescentes evaluados presentan desnutrición, el porcentaje restante se encuentra en un peso normal, el $36 \%$ superaron sin esfuerzo las pruebas físicas, el otro porcentaje $34 \%$ no superaron los test. Mientras que el $30 \%$ restante de deportistas cumplieron con las exigencias mínimas de los estándares establecidos por los test de aptitud física. Los adolescentes con buen IMC presentan mejor movilidad, mejor elasticidad y mejor respuesta a los trabajos de fuerza. Los adolescentes con un IMC bajo presentan mayor dificultad en las pruebas de velocidad con Conclusiones: El estado nutricional puede influir seriamente en la condición física y en consecuencia en el rendimiento deportivo del deportista.

Universidad de la Guajira, Riohacha, Colombia.

Palabras clave: índice de masa corporal, desnutrición, deporte.

\section{RENDIMIENTO OPERATIVO DIAGNÓSTICO DE LA HERRAMIENTA STRONGKIDS EN PACIENTES PEDIÁTRICOS HOSPITALIZADOS}

\section{Laura Cristina Sánchez Giraldo, Jennifer Tatiana Mappe, Olga Lucía Pinzón Espitia, Jairo Echeverry Raad}

Introducción: la prevalencia de desnutrición en población pediátrica hospitalaria oscila entre el 6 y el $50 \%$ en diferentes países, lo cual evidencia la necesidad de establecer acciones para cuantificar la prevalencia de riesgo o desnutrición al ingreso hospitalario en Colombia.

Objetivo: estimar la prevalencia del estado nutricional intrahospitalario utilizando patrones de referencia estandarizados y realizar la validación de criterio mediante la evaluación del rendimiento diagnóstico de la herramienta STRONGkids en niños hospitalizados en una entidad prestadora de salud de alta complejidad.
Métodos: estudio descriptivo de corte transversal en el que se seleccionaron pacientes pediátricos de forma consecutiva durante 5 semanas entre los meses de octubre y noviembre del 2018. La población final evaluada correspondió a 300 pacientes.

Resultados: la prevalencia de algún grado de desnutrición hospitalaria, fue del 3,5\% (IC 95\% 0.3-11) en menores de 5 años y 3,8\% (IC 95\% 0.8-10.6) en mayores de esa edad. STRONGkids, con un puntaje de 4 o mayor, mostró una Sensibilidad promedio del 81\% (IC 95\%, 40\%-100\%), y una especificidad promedio de $97.9 \%$ (IC 95\%, 94.5\%- 100\%) en el diagnóstico de desnutri- 
ción (aguda moderada, aguda severa, Delgadez), según los criterios antropométricos de la Resolución 2465 de 2016 del Ministerio de Salud. Razón de Verosimilitudes Positiva (RVP):39, Razón de Verosimilitudes Negativa (RVN):0.19, Razón de RVP/RVN: 205, a una probabilidad pre-prueba de Desnutrición en la serie del 5.6\%.
Conclusiones: STRONGkids es una herramienta válida en el diagnóstico de cualquier tipo de desnutrición hospitalaria en niños.

Palabras clave: desnutrición, tamización nutricional, riesgo nutricional, antropometría, STRONGkids.

Universidad Nacional de Colombia, Bogotá, Colombia.

\section{REFORMULACIÓN DE LA DIETA LÍQUIDA TOTAL SUMINISTRADA POR UN SERVICIO DE ALIMENTACIÓN HOSPITALARIO EN LA CIUDAD DE BOGOTÁ}

María Paula Guzmán Silva

Introducción: la alimentación del paciente hospitalizado es fundamental para la recuperación de su estado de salud, se ha determinado que el grado de aceptación de la dieta influye en la ingesta. Las dietas modificadas en textura, en especial la dieta líquida total, tienen baja aceptación por parte de los pacientes por sus características sensoriales.

Objetivo: reformular la dieta líquida total ofrecida por el servicio de alimentación de una institución hospitalaria de tercer nivel, teniendo un enfoque de calidad nutricional y sensorial. Fue un estudio observacional con prueba de análisis sensorial. Se evaluó la ingesta de 33 pacientes con dieta líquida total, se reformuló la minuta patrón y el ciclo de menús, además de realizar análisis sensorial de 6 menús ofertados por el servicio y 10 menús propuestos en la reformulación a 26 pacientes. Para el análisis de resultados de la evaluación de ingesta se empleó estadística descriptiva; para el análisis sensorial se utilizó análisis de correspondencias.

Resultados: se encontró que los pacientes con prescripción de dieta líquida total consumen el $50 \%$ de la alimentación ofrecida por el servicio, esto influenciado por un desagrado hacia las preparaciones. Los pacientes presentan un bajo nivel de aceptación por la dieta suministrada en el servicio, al realizar análisis con las preparaciones propuestas, se encontró una mayor aceptación por parte de los pacientes.

Conclusión: se evidenció una mejora en la aceptación de los pacientes por la dieta líquida total cuando esta se reformula con un enfoque en la calidad sensorial y nutricional.

Palabras clave: dieta liquida total, reformulación, innovación, fortificación.

Pontificia Universidad Javeriana, Bogotá, Colombia

\section{PROPUESTA DE PROTOCOLO PARA EL SOPORTE NUTRICIONAL EN PACIENTE HOSPITALIZADO CON DIAGNÓSTICO DE CÁNCER PARA EL INC E.S.E.}

Laura Fernanda Ortíz Ruíz, Ilvar José Muñoz Ramírez

Introducción: el cáncer es considerado hoy en día una de las causas de mor bilidad y mortalidad más importantes en todo el mundo, y la desnutrición resulta ser una de las características más comunes en este tipo de pacientes, siendo considerada como un signo de mal pronóstico, al reducir la efectividad del tratamiento y estar asociada a una disminución de la supervivencia.

Objetivo: establecer recomendaciones que aborden los aspectos clínicos correspondientes a la tamización, diagnóstico y tratamiento nutricional de pacientes adultos hospitalizados con diagnóstico de cáncer.

Métodos: se llevó a cabo una revisión narrativa de la más reciente literatura complementada con la opinión de expertos, médicos, nutricionistas, enfer-

Universidad Nacional de Colombia. Bogotá, Colombia

lafortizru@unal.edu.co, ijmunozr@unal.edu.co meros y químicos farmacéuticos del Instituto Nacional de Cancerología ESE y la Universidad Nacional de Colombia; desarrollada bajo un acercamiento a la metodología de Panel Delphi modificado.

Resultados: se definieron 4 secciones generales para un total de 14 recomendaciones y 1 tabla de decisión, que pretenden contribuir a la reducción de complicaciones prevenibles y mejoras en la calidad de vida de los pacientes del INC, por medio de la disminución de la variabilidad en la práctica clínica.

Palabras clave: nutrición clínica, cáncer, cirugía, nutrición enteral, pacientes críticamente enfermos.

\section{FACTORES DETERMINANTES EN EL ESTADO NUTRICIONAL EN NIÑOS CON PARÁLISIS CEREBRAL INFANTIL ESPÁSTICA}

Diana Fernanda Quesada Poveda, Natalia Caicedo Trujillo, Sayda Milena Pico Fonseca, Isabel Cristina Rojas Padilla

Introducción: la parálisis cerebral infantil se considera un trastorno del adecuado desarrollo psicomotor del niño, siendo la causa más frecuente de discapacidad motora en los infantes. El estado nutricional de estos pacientes es esencial para su estado de salud, por esto el soporte nutricional actualmente es considerado un factor muy importante para el adecuado manejo y seguimiento nutricional.

Metodología: el diseño de estudio de esta investigación es de tipo corte transversal, donde las fuentes de información para el caso de los menores con parálisis espástica serán de datos secundarios obtenidos de historia clínica y en el caso de los cuidadores se aplicará una encuesta para conocer las prácticas en el manejo de la alimentación del niño.

Programa nutrición y dietética escuela nacional del deporte, Bogotá, Colombia.
Resultados: el sexo masculino predominó con un $64 \%$ y el sexo femenino fue del $36 \%$. El estado nutricional de los infantes arrojó que el $72 \%$ de la muestra total los niños se encontraron con déficit, el $20 \%$ con exceso y tan solo el $8 \%$ en adecuado. Como resultados estadísticamente significativos para este trabajo, encontramos que el estado nutricional influye en los días de hospitalización pues se obtuvo un p-valor de 0,039, al igual que ¿por qué los niños utilizan la fórmula? Con un p-valor de 0,003 , por último, si los niños contaban con hospitalización en casa, con un p-valor de 0,04.

Conclusión: existen múltiples factores que logran determinar el estado nutricional en los niños que sufren de parálisis cerebral espástica.

Palabras clave: estado nutricional, parálisis cerebral espástica, menores de edad. 


\section{SEDENTARISMO Y CONSUMO DE ALIMENTOS ENTRE ESTUDIANTES DE DIFERENTES ÁREAS DE LA SALUD: UN ESTUDIO CROSS-SECTIONAL}

Angie Katherine Pérez Hoyos, Lina María Mosquera Rentería, Diana Paola Cala Liberato, María Camila Quiñones Sánchez

Introducción: los hábitos alimentarios y actividad física pueden ser diferentes entre los estudiantes de programas del área de la salud.

Objetivo: identificar las diferencias de los niveles de actividad física y el consumo de grupos de alimentos entre estudiantes de programas del área de la salud de la Universidad El Bosque.

Métodos: estudio transversal analítico que involucró estudiantes de pregrado de enfermería, optometría, medicina, odontología e instrumentación quirúrgica y estaban matriculados en I-IV semestre durante agosto del 2017. Mediante encuesta autodiligenciada se midió actividad física (IPAQ-SF) y hábitos alimentarios (cuestionario de frecuencia de consumo). Se emplearon las pruebas $\mathrm{Ji}^{2}$ y Kruskal-Wallis para las comparaciones entre programas.

Resultados: participaron 692 sujetos y $77.7 \%$ fueron mujeres. El $85.3 \%$ de los estudiantes desayunan y enfermería presenta el menor consumo $(80 \%$; p0.0092). Los lácteos son el grupo de alimentos que más se consume (47.1\%), existe un bajo consumo de verduras en toda la población (19.6\%) y es marcado en enfermería ( $15.6 \%$ p0.074). El 51\% de la población realiza actividad física y el nivel alto de actividad física se observa en hombres ( $p 0.001)$, en estudiantes de primer año y en medicina $(p<0.05)$.

Conclusiones: se encontraron diferencias en el consumo de alimentos y actividad física entre los programas. Los estudiantes de enfermería son más sedentarios y consumen en menor proporción el desayuno respecto a los otros programas. Los estudiantes de medicina presentaron mayor porcentaje de actividad física alta y tienen un consumo de alimentos similar a los demás programas.

Palabras claves: ejercicio, hábitos alimentarios, hábitos saludables, estudiantes del área de la salud, enfermedades crónicas.

Universidad El Bosque, Bogotá, Colombia.

\section{EFECTO DE LA SUPLEMENTACIÓN CON ANTIOXIDANTES SOBRE INDICADORES HEMATOLÓGICOS DURANTE EL TRATAMIENTO ONCOLÓGICO EN MUJERES CON CÁNCER CÉRVICO}

Maria Dolores Arias Soberón, Karolina Álvarez-Altamirano, Vanessa Fuchs-Tarlovsky.

Introducción: el cáncer cérvico uterino ocupa el 4o lugar en prevalencia en mujeres a nivel mundial; en México representa la segunda causa de morbilidad y mortalidad hospitalaria entre las mujeres después del cáncer de mama, lo que lo convierte en un problema de salud pública.

Objetivo: conocer el efecto que tiene la suplementación con antioxidantes a diferentes dosis sobre indicadores hematológicos (hemoglobina e índices hematimétricos) durante el tratamiento antineoplásico con cisplatino y radioterapia.

Materiales y métodos: ensayo clínico aleatorizado, ciego, prospectivo, longitudinal, realizado en el Hospital General de México en pacientes diagnosticadas con cáncer cérvico uterino con tratamiento quimioterapéutico de cisplatino exclusivo a dosis de $40 \mathrm{mg} / \mathrm{m}^{2}$ y radioterapia a dosis de $50 \mathrm{~Gy}$ en 25 sesiones. Las pacientes se dividieron aleatoriamente en tres grupos: placebo (PP), grupo con una cápsula de antioxidantes (SP), y grupo con dos cápsulas de antioxidantes (SS); cada cápsula de antioxidante contiene: B caroteno $400 \mathrm{mcg}$, Vitamina E 200mg, Vitamina C $200 \mathrm{mg}$, Selenio 50mcg y Zinc $15 \mathrm{mg}$. Se realizaron pruebas de biometría hemática $(\mathrm{BH})$, medición de peso corporal y aplicación de frecuencia de consumo de alimentos al inicio y al final del tratamiento oncológico.
Resultados: completaron su tratamiento 52 pacientes: grupo $\mathrm{PP}=10$, grupo $\mathrm{PS}=16$ y grupo $\mathrm{SS}=26$. Solamente el grupo SS no presentó cambios significativos en los niveles de hemoglobina antes y después del tratamiento con un valor promedio final de $\mathrm{Hb}=11.51 \mathrm{~g} / \mathrm{dL} \pm 1.7$ ( $\mathrm{p}=0.053$ ); este mismo grupo presenta un incremento significativo en las concentraciones de $\mathrm{HbCM}$ $(p=.000), C M H b C(p=.011)$ y VCM $(p=0.004)$; para el caso del grupo SP presentó aumento significativo en $\mathrm{HbCM}(\mathrm{p}=.001)$ y $\mathrm{CMHbC}(\mathrm{p}=.029)$. El grupo no suplementado (placebo) no presenta cambios significativos en $\mathrm{HbCM}, \mathrm{CMHbC}$, ni VCM. Para las concentraciones de hematocrito y eritrocitos los tres grupos disminuyen de manera significativa $(p<0.05)$. Los cambios en la alimentación y el peso corporal disminuyeron significativamente en todos los grupos. El IDR de vitamina E y Selenio no se alcanza en ningún grupo con la dieta habitual.

Conclusiones: la suplementación con antioxidantes ayuda a mantener estables los niveles de $\mathrm{Hb}$ en sangre, lo cual disminuye el riesgo de ser transfundidas. Hay una disminución significativa en la ingesta y el peso corporal de las pacientes. Hay mala variedad alimentos en la dieta.

Palabras clave: antioxidantes, hemoglobina, cáncer cérvico uterino.

Hospital General de México, México. 


\title{
Resúmenes Premio José Félix Patiño Restrepo
}

\author{
TRABAJOS LIBRES
}

\begin{abstract}
EFECTO DE LA INGESTA DE SOYA SOBRE LA INFLAMACIÓN EN EL EJERCICIO FÍSICO EXHAUSTIVO EN MODELO ANIMAL
\end{abstract}

Diana Aguirre Rueda, Rubén Darío Pulgarín, Diana Cecilia Álvarez Salazar

Introducción: el ejercicio exhaustivo está asociado a la activación inflamatoria. El uso excesivo de antiinflamatorios no esteroideos en deportes está relacionado con el deterioro de varios sistemas. Los flavonoides constituyen propiedades antiinflamatorias.

Objetivo: determinar el efecto de la soya sobre niveles de inflamación producidos por ejercicio físico exhaustivo en plasma y músculo.

Métodos: 30 ratas machos Sprawley dawley (180-200 g de peso), divididos en grupo sedentario sin intervención (C), ingesta de soya a prueba $(E+T P)$ y sin ingesta de soya a prueba (E). Para inducir inflamación se aplicó el Morris Water Maze Test (Morris, 1981). Marcadores inflamatorios TNF-a, IL-1 $\beta$ e IL-6 en plasma mediante ELISA y COX-2 e iNOS en cuádriceps mediante Western-blot.
Marcador antiinflamatorio PPAR- $\gamma$. Los valores se expresan como media $\pm D . S$. Las diferencias entre los grupos se evaluaron mediante ANOVA. Los experimentos se realizaron bajo el cumplimiento de la normativa y aprobación del comité ético de la Universidad de Valencia, España.

Resultados: Disminución en plasma de niveles inflamatorios en grupo suplementado con soya y aumento de PPAR- $\gamma$.

Conclusiones: compuestos naturales como la soya disminuye niveles inflamatorios causados por ejercicio físico exhaustivo y aumentan el marcador antiinflamatorio generando una acción beneficiosa.

Palabras clave: inflamación, soya, antiinflamatorio, ejercicio exhaustivo.

Facultad de Cultura Física, Deporte y Recreación, Universidad Santo Tomás, Bucaramanga, Colombia. Universidad Industrial de Santander

\section{ASOCIACIÓN ENTRE FUERZA PRENSIL DE MANO Y MARCADORES DE RIESGO CARDIOMETABÓLICO EN UNA COHORTE DE ADULTOS ECUATORIANOS}

Ludwig Álvarez C, Vanessa Tapia M, Lissette Santamaría M, Johnny Galvez C, Cecilia Arteaga P.

Introducción: la fuerza muscular adecuada se asocia con menor riesgo cardiometabólico. La obesidad, dislipidemia, hipertensión e hiperglicemia en ayunas se identifican como factores de riesgo cardiometabólico.

Objetivos: evaluar la asociación entre fuerza prensil de mano (FPM) y marcadores de riesgo cardiometabólico (MRC) y establecer su correlación.

Métodos: estudio descriptivo y transversal, se evaluaron a 126 participantes. Las variables dependiente fue: FPM, y las independientes: circunferencia de cintura, glicemia, colesterol HDL, triglicéridos y presión arterial. Se usó regresión logística variable para obtener la relación entre FPM y MRC.

Universidad Católica de Santiago de Guayaquil, Ecuador.
Resultados: del total de 126 adultos, de edad media de 39,1 \pm 7,2 años, el $72,2 \%$ fueron mujeres y el $27,8 \%$ hombres. La FPM tiene una correlación positiva con el peso corporal $(r=0,433 ; p=0,021)$ y $c c(r=0,190 ; p=0,033)$. Correlaciones inversas fueron encontradas entre la fuerza prensil de mano $y$ el colesterol HDL $(r=-0,203 ; p=0,022)$.

Conclusiones: la FPM alta se asocia a un perfil metabólico favorable con un menor riesgo de marcadores cardiometabólicos. La medición de FPM relativa es un factor predictivo para un riesgo de enfermedad metabólica que la medición absoluta de FPM.

Palabras clave: fuerza prensil, riesgo cardiometabólico, obesidad.

\section{DEUDA CALÓRICA Y PROTEICA EN PACIENTES NEUROCRÍTICOS EN MANEJO CON SOPORTE NUTRICIONAL ENTERAL, PARENTERAL O MIXTO}

Leidy Marcela Romero Vargas

Introducción: el paciente neurocrítico requiere soporte nutricional temprano dado el alto grado de catabolismo al que se enfrenta; la principal vía elegida es la gastrointestinal, donde se precisa alcanzar metas calóricas y proteicas propuestas; sin embargo, en ciertas ocasiones no se logra este objetivo dado frecuentes interrupciones en el manejo nutricional instaurado, lo cual conlleva a ciertas complicaciones.

Objetivo: describir y comparar los resultados del alcance de meta calórica y proteica propuesta en pacientes con patología neurocrítica admitidos en la UCl del Hospital Universitario Mayor-Méderi para diferentes desenlaces clínicos.
Metodología: se incluyen pacientes mayores de 18 años de edad admitidos en UCI por patología neurológica; se realiza valoración nutricional de forma subjetiva. Se inicia soporte nutricional precoz con el fin de lograr hacia el 2 día de manejo meta calórica estimada (con ecuación de penn state) y meta proteica (no inferior al 15\% de los requerimientos estimados). Se revisa de forma diaria los registros de enfermería; si se observan interrupciones se indaga motivo de suspensión.

Resultados: se obtuvo una muestra de 29 pacientes neurocríticos; 8 pacientes con deuda calórica y proteico vs 21 pacientes sin la misma. Frente a 
desenlaces clínicos se obtuvo mayor mortalidad, días de soporte ventilatorio (entre 1 a 5 días) y estancia en $\mathrm{UCl}$ (entre 1 a 10 días) en pacientes con deuda calórica y proteica.
Conclusión: el soporte nutricional de inicio temprano, sin interrupciones frecuentes, es de vital importancia para reducir la deuda calórica y proteica que repercuten negativamente en la evolución clínica del paciente neurocrítico.

Palabras clave: soporte nutricional, paciente neurocrítico, deuda calórica, deuda proteica, requerimiento calórico y proteico.

Hospital Universitario Mayor-Méderi. Bogotá, Colombia.

\section{RIESGO CARDIOMETABÓLICO Y MASA GRASA CORPORAL EN NIÑOS Y ADOLESCENTES DE BUCARAMANGA, COLOMBIA}

Norma Serrano 1,23, Diana Paola Suárez, Adriana Robles Silva4, Edna Magaly Gamboa-Delgado5, Doris Cristina Quintero-Lesmes'.

Introducción: en niños y adolescentes, la obesidad se asocia con factores de riesgo cardiometabólicos como hipertensión, hiperlipidemia, resistencia a la insulina y diabetes tipo 2, que pueden conducir a enfermedades cardiometabólicas en la edad adulta. Esta asociación implica la necesidad de identificar métodos fáciles, de bajo costo y confiables para identificar individuos que están en mayor riesgo.

Objetivo: evaluar la asociación entre el porcentaje de grasa corporal y los factores de riesgo cardiometabólico en los niños y adolescentes de la ciudad de Bucaramanga.

Fundación Cardiovascular de Colombia, Floridablanca, Colombia

Hospital Internacional de Colombia, La Parcela, Piedecuesta, Santander

Fundación Universitaria, La Parcela, Piedecuesta, Santander

Unión Temporal CARDIECOL Universidad Autónoma de Bucaramanga-UNAB, Floridablanca, Colombia

5 Escuela de Nutrición y Dietética, Universidad Industrial de Santander-UIS, Bucaramanga, Colombia.

dorisquintero@fcv.org
Métodos: se estudiaron 494 niños y adolescentes jóvenes entre los 10 y< de 20 años de edad; se les tomó pruebas de laboratorio para análisis de marcadores de riesgo cardiovascular y medidas antropométricas. El porcentaje de grasa corporal fue medido con la ecuación de Slaughter. Se realizaron análisis de regresión lineal para evaluar la asociación entre los factores de riesgo cardiometabólicos y el porcentaje de grasa corporal.

Resultados: la prevalencia de porcentaje de masa grasa corporal ( $>26 \%$ ) fue de $46,1 \%$. Las variables con las que se asoció significativamente el porcentaje de grasa corporal fueron la resistencia a la insulina Rl, los niveles de colesterol HDL y LDL, triglicéridos, colesterol total e hipertensión arterial.

Conclusiones: el incremento en el porcentaje de grasa corporal se asocia significativamente con los factores de riesgo cardiometabólicos en niños, adolescentes de Bucaramanga. La identificación e intervención temprana de esta población en riesgo es primordial.

Palabras claves: grosor de los pliegues cutáneos, factores de riesgo, enfermedad cardiovascular, resistencia a la insulina.

\section{CONCENTRACIÓN DE ÁCIDOS GRASOS DE CADENA CORTA EN PACIENTES CRÍTICOS CON SEPSIS Y VOLUNTARIOS SANOS}

Gloria María Agudelo Ochoa', Nubia Amparo Giraldo', Ana María Jaillier², Adriana Giraldo Villa ${ }^{3}$, Irene Acevedo ${ }^{4}$, Monoica Yepes $^{5}$, Janeth Barbosa ${ }^{6}$, Carlos Barrera7, Beatriz Helena Valdés ${ }^{8}$

Objetivos: comparar la concentración de ácidos grasos de cadena corta (AGCC) en pacientes críticos con sepsis (PCS) y voluntarios sanos (VS).

Métodos: estudio observacional descriptivo, multicéntrico en unidades de cuidados intensivos de cinco instituciones. Muestra conformada por pacientes críticos con sepsis ingresados a Unidades de Cuidados Intensivos durante 6 meses que cumplieron criterios de inclusión; por cada paciente, se reclutó un voluntario. De cada sujeto se recolectó una muestra espontánea de heces; la información de las variables de interés de los PCS fue obtenida

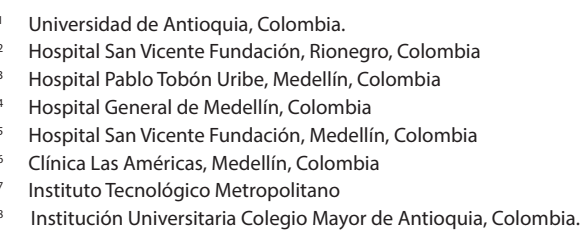

de la historia clínica. Para determinar la concentración de los AGCC se utilizó un cromatógrafo de gases acoplado a espectrómetro de masas (Agilent 7890/ MSD 5975C).

Resultados: muestra final de 44 PCS y 45 VS. La distribución por edad y sexo no mostró diferencias entre los grupos ( $p>0.05$ ); por IMC, se encontró mayor desnutrición en los PCS $(P=0.024)$. La concentración total y de los 4 AGCC evaluados (Propiónico, acético, butírico, isoburtírico) fue significativamente menor en los PCS respecto a los VS $(p=0.000)$. En los PCS, no se halló asociación con variables desenlace; por diagnóstico, se encontró disminución significativa en los ácidos butírico e isobutírico en los pacientes con diagnósticos de infección y enfermedad pulmonar al ingreso $(p<0.05)$.

Conclusiones: los resultados de este estudio confirman que en PCS la concentración de AGCC está significativamente disminuida comparada con VS. Aunque no se encontró asociación con variables desenlace, los hallazgos sugieren que su modulación podría ser importante en la terapia intestinal como parte de los protocolos de atención en $\mathrm{UCl}$.

Palabras clave: paciente crítico, sepsis, ácidos grasos de cadena corta, unidad de cuidados intensivos.

\section{EFECTO DEL 6-SHOGAOL SOBRE LA CAPTACIÓN DE GLUCOSA Y SUPERVIVENCIA DE CÉLULAS TUMORALES DERIVADAS DE FIBROSARCOMA HUMANO (HT1080)}

Angie Carolina Romero Arias, Andrés Felipe Aristizabal Pachón, Ludis del Rosario Morales Álvarez

Introducción: el jengibre es una planta nativa del sur de China y en la última década la investigación sobre sus compuestos ha aumentado significativamente. Dentro de sus componentes, el 6-shogaol es, posiblemente, uno de los compuestos con mayor capacidad antitumoral. Sin embargo, el mecanismo molecular a través del cual el 6-shogaol ejerce su efecto aún no se ha dilucidado. 
Objetivo: este trabajo evaluó el efecto del 6-shogaol sobre la captación de glucosa y la supervivencia de células tumorales derivadas de fibrosarcoma humano (HT1080). La viabilidad de células tumorales (HT1080) y células normales (fibroblastos) se determinó mediante el ensayo MTT con 18 concentraciones diferentes de 6-shogaol (2,5-150 $\mu \mathrm{M})$. Posteriormente, se midió el efecto del 6-shogaol en la producción de ROS, la captación de glucosa y la expresión de proteínas de la vía de señalización PTEN / Akt / mTOR.

Resultados: el 6-shogaol ejerce un efecto citotóxico en las células tumorales asociado al aumento en la producción de ROS. Efecto diferencial fue encontrado en los fibroblastos. La $\mathrm{N}$-acetilcisteína disminuyó la producción de ROS y recuperó la viabilidad celular. Asimismo, la captación de glucosa disminuyó con las concentraciones incrementales de 6-shogaol y aumentó la expresión de mTOR-p y Akt-p; PTEN fue activo en todos los tratamientos con 6shogaol.

Conclusiones: aumentar la producción de ROS por el 6-shogaol se asocia con la muerte celular; probablemente, debido a la disminución de uno de los principales nutrientes para las células tumorales como la glucosa, que junto con la activación de la vía de señalización Akt pueden inducir la senescencia celular.

Palabras clave: fibrosacoma, 6-shogaol.

Pontificia Universidad Javeriana. Bogotá, Colombia.

\section{EFECTO DE UN PROGRAMA DE EJERCICIO ESTRUCTURADO SOBRE INDICADORES DE RIESGO CARDIOVASCULAR EN ADULTOS MAYORES DE BOGOTÁ}

Barón Jaime', Rodríguez Leandro', Carrillo Cristian', Valencia Omaira²

Objetivo: evaluar el efecto de un programa de ejercicio dirigido a una población de adultos mayores sobre indicadores de riesgo cardiovascular, clínicos, bioquímicos y antropométricos.

Métodos: un grupo de adultos mayores asistentes al programa de crónicos del hospital del Sur en Bogotá fue asignado de manera aleatoria a dos grupos de intervención El primer grupo continuó su asistencia al programa de cró nicos (grupo control), y con el segundo grupo (grupo intervención) se inició un programa de ejercicio estructurado con 3 sesiones semanales de 1 hora durante 14 semanas. La medición de factores de riesgo cardiovascular incluyó indicadores bioquímicos como colesterol total y HDL, triglicéridos, glicemia y creatinina; indicadores clínicos se incluyeron la presión arterial y la frecuencia cardiaca. Como indicadores antropométricos se evaluaron el índice de masa corporal (IMC), porcentaje de grasa, masa magra y masa muscular usando medidor de composición corporal marca SECA. Estas mediciones se realizaron al inicio (T0) y al finalizar la intervención (T1). El efecto del programa se evaluó a través de un análisis de Wilcoxon para determinar cambios entre T0 y T1. Se realizó una regresión lineal de los cambios no esperados y su asociación con factores sociodemográficos.

SENA, Área de actividad física y Nutrición. Bogotá, Colombia.

Universidad de los Andes, Bogotá, Colombia.
Resultados: se incluyeron 63 participantes ( $82.5 \%$ mujeres, con una media de edad de 61.2 años, desviación estándar (DE) \pm 5.6 ). 42 participantes fueron asignados al grupo intervención. Se encontró una disminución significativa (Dif T0-T1) de la presión arterial sistólica $(4.2 \mathrm{mmH})$, y triglicéridos (3.1 mg) respecto a la línea de base; por el contrario, se encontró un aumento significativo de los niveles de glicemia $(5.8 \mathrm{mg})$, colesterol total $(5.8 \mathrm{mg}$ ) y HDL (3.8 mg) $(p<0.005)$. No se encontraron cambios significativos en las medidas antropométricas entre T0 y T1, IMC ( $p=0.068)$, masa grasa $(p=0.823)$, masa muscular ( $p=0.128)$ y grasa visceral $(p=0.980)$. Se evidenció una disminución significativa de masa magra dif T0 $-\mathrm{T} 1=1$ punto porcentual $(\mathrm{p}<0.005)$. Se encontró un efecto positivo de los participantes cuya ocupación es el hogar sobre los niveles de glicemia ( $p=0.032)$; también se encontró un efecto positivo entre el sexo (masculino) y la masa magra $(p=0.010)$.

Conclusiones: el programa de ejercicio estructurado en la población de adultos mayores es una estrategia prometedora para mejorar los factores de riesgo cardiovascular bioquímicos y clínicos, no se encontraron cambios significativos en las medidas de composición corporal a excepción del cambio en masa magra. Para una medición efectiva de los efectos del programa, hace falta incluir evaluación de consumo de alimentos e intervenciones en alimentación y nutrición a fin de determinar su impacto en el cambio de dichos indicadores.

Palabras clave: actividad física, adulto mayor, riesgo cardiovascular, ejercicio.

\section{MONITORÍA METABÓLICA EN NEONATOS CRÍTICAMENTE ENFERMOS CON NUTRICIÓN PARENTERAL, FLORIDABLANCA, COLOMBIA}

Jorge Alvarado Socarras

Introducción: la nutrición parenteral es necesaria durante el periodo neonatal, sin embargo esta no está libre de efectos adversos. Algunos de estos efectos adversos están relacionados con la morbimortalidad, como son las infecciones y la aparición de colestasis.

Objetivo: describir la monitoria metabólica de los neonatos sometidos a nutrición parenteral y evidenciar si existe algún grupo poblacional y patologías relacionadas, con las alteraciones detectadas.

Metodología: se realizó un estudio de corte transversal en 25 neonatos sometidos a nutrición parenteral en una institución de salud de alto nivel de complejidad, donde se evalúo el perfil metabólico cada 15 días en un periodo de tres meses. Resultados: se encontró que la bilirrubina directa se alteró en
61\% de los neonatos los primeros 15 días de nutricional parenteral, seguida de alteraciones en SGPT, SGOT, proteínas, potasio y bilirrubina indirecta.

Conclusiones: por lo reportado, va a existir un grupo de pacientes de mayor riesgo de complicaciones hepáticas, por lo que se decidió individualizar la monitoria de rutina en el grupo de neonatos expuestos a nutrición parenteral. Hoy con la aparición de mezclas de aminoácidos y lípidos, con composiciones más seguras, la posibilidad de colestasis, se disminuye al menos de forma temprana (3-4 semanas). La monitoria debe realizarse, pero se necesitan más estudios que permitan determinar el tiempo para realizarlas de forma rutinaria o si la mejor manera es individualizar dependiendo de los factores de riesgo.

Palabras claves: nutrición parenteral, colestasis, recién nacido prematuro. 


\section{CONCORDANCIA ENTRE GLICEMIA CENTRAL Y GLUCOMETRÍA EN PACIENTES CON NUTRICIÓN ARTIFICIAL CONTINUA EN UNA UNIDAD DE CUIDADO INTENSIVO DE ADULTOS}

\section{Walter Villalobos}

Introducción: los pacientes con nutrición artificial requieren monitoreo de los niveles de glucosa plasmática que puede obtenerse entre 45 a 120 minutos por vía venosa y entre 15 a 30 segundos por vía capilar. La diferencia entre los dos métodos oscila entre el $8 \%$ y el $20 \%$. La glucometría en el paciente crítico se utiliza en Colombia como una medida confiable, no obstante, su alta variabilidad con respecto a la glicemia central.

Objetivo: estimar la concordancia y la diferencia relativa de la media absoluta (MARD) entre la glucometría y la glicemia central en pacientes críticos con soporte nutricional continuo.

Métodos: estudio de concordancia prospectivo por método de blandaltman y coeficiente de correlación concordancia (CCC).

Ámbito: unidad de cuidados intensivos de adultos.
Resultados: cincuenta y seis pacientes ingresaron al estudio de los que $23(41,1 \%)$ eran hombres; cincuenta $(89,3 \%)$ recibieron nutrición enteral. Se obtuvieron 196 pares de muestras totales de glicemia central y glucometría, de las que 140 fueron después de iniciar nutrición. La MARD fue 8,55\% (RIQ $11,24 \%)$. El CCC fue más alto en ayuno 0,96 (IC 95\% 0,94-0,98) que el postprandial 0,84 (IC 95\% 0,77-0,88).

Conclusión: hay una buena, pero no suficientemente precisa concordancia entre glicemia y glucometría en pacientes críticos con nutrición artificial. No se sugiere el uso rutinario de glucometría como única medida en Unidad de Cuidados Intensivos.

Palabras clave: Glicemia, nutrición parenteral, nutrición enteral, cuidados críticos, coeficiente, correlación, concordancia.

Clínica Universidad de la Sabana, Chía, Colombia.

\section{PATRONES ALIMENTARIOS Y CÁNCER DE MAMA EN COLOMBIA}

Objetivo: establecer la asociación entre los patrones de consumo dietario y la incidencia de cáncer de mama. Además, predecir la incidencia a nivel ecológico con base en variables biológicas y socioeconómicas.

Métodos: se analizaron datos para 24 departamentos de Colombia. En ellos habitaban 13.941 .000 mujeres ( $95 \%$ del total las mujeres entre 18 y 54 años en 2010). Mediante regresión lineal, se estableció la asociación entre la incidencia de cáncer de mama, 2007-2011, y los patrones de consumo dietario, 2010. Adherencia al consumo en tres patrones dietarios (puntajes Z); fibra/ lácteos, tradicional/almidón y snack. Además, veintiún variables del contexto. La asociación entre incidencia de cáncer (Main outcome) y los patrones dietarios, se estableció con coeficientes $\beta$, estandarizados, crudos y ajustados. Los patrones de consumo dietario se derivaron de análisis factorial de la Encuesta Nacional de Nutrición, 2010. La información sobre el contexto social y económico fue obtenida de fuentes oficiales.

Resultados: para el patrón tradicional/fibra el $\beta=-30467$ (IC 95\%: -58282 a $-2651 ; P<0,001)$, para el snack de $\beta=-43612$ (IC95\%: -78220 a $-9004 ; P=0,016)$. La bondad de ajuste para el modelo es de $R^{2}=84 \%$. La correlación entre la incidencia de cáncer observada y la estimada fue de $r=0,92$ (IC 95\%: 0,92 a 0,97). El estadístico Bland y Alman de -0,49 (IC 95\%: -5,60 a 5,70).

Conclusión: los patrones, tradicional/almidón y snack la tasa de analfabetismo y la duración de la lactancia materna, están asociados a la incidencia de cáncer de mama.

Palabras clave: cáncer de mama, lactancia materna.

Universidad Industrial de Santander. Fundación Cardiovascular de Colombia

\section{CORRELACIÓN ENTRE EL CONSUMO DE ÁCIDOS GRASOS EN MUJERES LACTANTES Y SU CONTENIDO EN LA LECHE MATERNA MADURA}

Beatriz Estella Marín

Introducción: estudios muestran dependencia entre el consumo de Ácidos Grasos (AG) y su contenido en la leche materna madura (LMM), incluyendo el contenido del ácido ruménico (AR) un CLA con un importante papel en la salud del lactante.

Objetivo: determinar la correlación entre el consumo de AG de la madre con la composición de estos en la LMM.

Metodología: en $30 \mathrm{ml}$ de LMM se cuantificó el perfil de AG bajo el método 996 de la AOAC en un cromatógrafo Agilent 6890B. El consumo de AG se hizo bajo cuestionarios de frecuencia y consumo habitual, la determinación del contenido de AG en cada alimento se hizo por Tablas de Composición de Alimentos.

Universidad de Antioquia, Medellín, Colombia.
Resultados: el 100\% de las madres no cumplen con las recomendaciones de ARA, w6 y w3, seguido por un alto porcentaje de madres con bajo consumo de DHA (86\%), EPA (82\%), AGM (82\%) y AGPI (70\%). El perfil de AG en la LMM mostró: las grasas saturadas tuvieron el mayor contenido $2,05 \pm 0,77$ $\mathrm{g} / 100 \mathrm{ml}$, en los poliinsaturados el contenido fue de $1,77 \pm 0,851 \mathrm{~g} / 100 \mathrm{ml}$, el contenido de insaturados fue de $3,36 \mathrm{~g} / 100 \mathrm{ml}$, en promedio el contenido de AR fue de $55 \%$ del total de los CLA.

Conclusión: no hay correlación entre el consumo de AG y su contenido en la LMM, este parece estar dado principalmente por las necesidades nutricionales del bebé y está más directamente relacionado con un proceso fisiológico de la glándula mamaria que permite proporcionar los nutrientes en las cantidades adecuadas en la LMM.

Palabras clave: leche materna madura, ácidos grasos, ácido ruménico, lactante. 


\section{INSEGURIDAD ALIMENTARIA Y DESARROLLO HUMANO PREDICEN LA INCIDENCIA DE CÁNCER COLORECTAL EN COLOMBIA}

Óscar Fernando Herrán Falla, Diana Cecilia Álvarez Salazar, Carlos Fuentes Sandoval

Introducción: la inseguridad alimentaria (INSA) ha sido asociada a la generación de enfermedad crónica y como barrera en el tratamiento del cáncer. Ante la ausencia de estudios, establecimos a nivel ecológico la relación entre INSA y la tasa de incidencia de cáncer de colon, recto y ano x 100.000 habitantes (ICCRA).

Métodos y Materiales: estudio ecológico realizado con información secundaria que producen regularmente las agencias del estado y que miden el nivel de desarrollo humano, social y económico en el territorio en las 33 unidades geodemográficas que conforman a Colombia. Utilizando regresión lineal múltiple con la ICCRA como la variable dependiente, la INSA como principal explicatoria y otras variables del contexto, establecimos la relación entre INSA y la ICCRA y ecuaciones predictivas de la ICCRA por sexo. La bondad de

Universidad Industrial de Santander, Bucaramanga, Colombia. ajuste en los modelos se garantizó mediante el coeficiente de determinación $\left(R^{2}\right)$ y el método de Bland y Almant.

Resultados: la INSA se relaciona de manera inversa y el IDH de manera directa con la ICCRA. Dos ecuaciones predicen con un alto grado de precisión la ICCRA; En hombres, [ICCRA $=-6,38+-0,25 *$ INSA + 35,5* IDH]. En mujeres, [ICCRA $=6,47+-0,23 *$ INSA $+19,1 *$ IDH] . El coeficiente de determinación $R^{2}$ (\%) en hombres es de 88 y en mujeres de 72. La diferencia media entre ICCRA observada versus la estimada fue de $-0,00$ en hombres (IC 95\%: -3,13 a 3,13), y en mujeres de -0,00 (IC 95\%: -4,08 a 4,08).

Conclusión: la INSA puede estar en la vía causal de la ICCRA. La INSA y el IDH predicen con precisión la ICCRA.

Palabras clave: Neoplasias del colon, abastecimiento de alimentos, desarrollo humano.

\section{CARACTERIZACIÓN DE PACIENTES CRÍTICOS QUE RECIBIERON SOPORTE NUTRICIONAL EN UN HOSPITAL DE III NIVEL}

Sandra Milena Rojas Molina

Introducción: las necesidades nutricionales en los enfermos críticos no se comprenden bien y varían según la fase de la enfermedad crítica. Aunque los resultados no se han estudiado adecuadamente en los ensayos aleatorios, el objetivo principal del apoyo nutricional es alterar el curso y el resultado de la enfermedad crítica. La fase de recuperación que comienza a medida que se resuelve la enfermedad crítica se caracteriza por un anabolismo que excede el catabolismo.

Objetivo: caracterización de los pacientes críticos que recibieron soporte nutricional

Método: estudio descriptivo, observacional, retrospectivo. Datos analizados en SPSS versión 21.

Resultados: se incluyeron 542 pacientes, el 57\% correspondió a el sexo masculino, edad media de 56 años, rango entra 14 y 97 años, peso promedio

Universidad Surcolombiana. Neiva, Colombia
65 kg, el 79,2 \% recibieron nutrición enteral y el 3,1\% nutrición mixta, vía de administración: sonda nasogástrica $57,7 \%$, gastrostomía $6,8 \%$ y sonda orogástrica $16,4 \%$, el $14,2 \%$ recibieron formulas poligoméricas, $5,1 \%$ oligoméricas y 80,7\% especiales, días de alimentación mínimo 2 máximo 187 días, glicemia máxima 502 media de 134, creatinina máxima de 23,3, media de 1,3, se realizó prueba de chi cuadrado encontrando una relación estadísticamente significativa entre vía de alimentación y días de alimentación con una p de 0,000, uso de inotrópico y mortalidad p 0,000.

Conclusiones: el soporte nutricional continua siendo una herramienta fundamental para pacientes críticos ayudando a la modulación de su respuesta inflamatoria, así mismo la adecuada elección de soporte nutricional hace la diferencia a largo plazo.

Palabras clave: soporte nutricional, paciente crítico.

\section{CONSUMO DE FRUTAS Y VERDURAS Y ESTADO NUTRICIONAL EN ADOLESCENTES ESCOLARIZADOS DE UN MUNICIPIO DEL CARIBE COLOMBIANO}

Introducción: existe relación entre el consumo de frutas y verduras (CFV) y el estado nutricional (EN); aunque en estudios previos se ha indagado por el consumo de estas, no se ha relacionado esto con indicadores antropométricos en el caribe colombiano.

Objetivo general: determinar la relación entre CFV y EN en adolescentes escolarizados de un colegio de Soledad, Atlántico, durante el año 2018.

Métodos: estudio Descriptivo. Participaron 260 adolescentes de 14-18 años. Se aplicó una encuesta de 3 módulos: sociodemográfico, frecuencia de CFV (estandarizado según ENSIN 2015), y variables: peso, talla y perímetro de cintura. Se procesó la información a través del Anthro Plus (OMS) empleando puntos de corte establecidos por la resolución 2465 de 2016.

Resultados: no se encontró asociación $(p>0,05)$ entre CFV y talla para la edad ( $p>0,05$ ); la mayoría de adolescentes presentaron talla adecuada para la

Universidad del Atlántico, Barranquilla, Colombia. edad, en porcentajes que van del $57,1 \%$ al $80,9 \%$. Tampoco se encontró asociación ( $p>0,05)$ entre CFV e IMC ( $p>0,05)$, ya que tanto en los que consumían $400 \mathrm{gr}$ o más o menos de $400 \mathrm{gr}$, la mayoría tenía un IMC adecuado para la edad, en porcentajes que van del $53,6 \%$ al $79,6 \%$ y no se encontró asociación $(p>0,05)$ entre en CFV y el índice cintura/talla $(p>0,05)$.

Conclusión: para determinar el estado nutricional de los adolescentes es necesaria la evaluación de indicadores que permitan determinar factores relacionados con malnutrición. El CFV es un indicador importante para mantener una buena calidad de vida y EN adecuado, pero hay que correlacionar otras variables como consumo de alimentos hipercalóricos y con alto índice glicémico.

Palabras clave: frutas, verduras, estado nutricional, adolescentes. 


\section{PHARMACIST TRAINING IN ARTIFICIAL NUTRITION IN LATIN AMERICA}

Background: Artificial nutrition is one of the disciplines that are part of modern medicine worldwide generating great impact on patient's quality of life at the ambulatory and intrahospitalary level. Hence, the importance of the pharmaceutical chemist in the clinical nutrition programs and its incorporation in the pharmacy study plans and their equivalents in Latin America.

Objective: Systematize the information available on the teaching of nutrition at the level of pharmacy undergraduate programs in Latin America, including the current situation, perspectives / challenges and recommendations.

Methods: A structured review of studies on the teaching of nutrition in pharmacy programs in Latin America was made in PubMed, Scopus, Scielo and Google Scholar; without time limit. In addition, an internet search of study plans of the different pharmacy programs in this region was performed.
Results: Fifteen publications on nutrition education and related subjects in pharmacy programs were identified. In addition, information from 143 pharmacy study plans was obtained, of which $84.6 \%$ include education in nutrition and $38.5 \%$ are accredited programs. A total of ten countries, highlighted because, in all of its undergraduate pharmacy, was included the area of interest.

Conclusions: The information available in publications and pharmacy's study plans shows a growing interest in nutrition education and related subjects in Latin America. However, it's necessary that universities perform integrated work with health institutions, oriented to design and proper use of artificial nutrition. Likewise, encourage the dissemination of the results obtained.

Keywords: Clinical nutrition, Bromatology, Hospital pharmacy, Pharmaconutrition, Curriculum.

Grupo de Investigación Promoción y Prevención Farmacéutica. Departamento de Farmacia, Facultad de Ciencias Farmacéuticas y Alimentarias, Universidad de Antioquia, Medellín, Colombia. diana.cardona@udea.edu.co

\section{DISEÑO DE UN PROTOTIPO DE APLICACIÓN PARA TELÉFONOS MÓVILES INTELIGENTES ÚTIL EN NUTRIVIGILANCIA}

Edwin Osorio ${ }^{1,2}$, Angie Cepeda', Diana Cardona $^{2}$

Introducción: la Nutrivigilancia contribuye a la seguridad del consumidor de suplementos dietarios, alimentos fortificados y nutriciones artificiales (enteral y parenteral), mediante la identificación de posibles efectos adversos relacionados con su consumo. En Colombia y Latinoamérica poco se conoce acerca del tema, y mucho menos de una herramienta para el reporte de eventos adversos causado por estos productos.

Objetivo: diseñar un prototipo de aplicación para dispositivos móviles que permita el reporte de eventos adversos asociados a suplementos dietarios y nutrición artificial.

Metodología: se realizó una búsqueda libre de artículos relacionados con nutrivigilancia y estudios clínicos, publicados en inglés, francés y español, sin límite de fecha, con acceso a texto completo, en PubMed/MedLine y ScienceDirect. Con la información obtenida se elaboró una propuesta de

Grupo de Investigación Ciencia, Tecnología e Innovación en Salud (CITEISA) SENA. Medellín, Colombia.

2 Grupo de Investigación Promoción y Prevención Farmacéutica, Facultad de Ciencias Farmacéuticas y Alimentarias, Universidad de Antioquia; Medellín, Colombia. jair.osorio@udea.edu.co formato de reporte de nutrivigilancia. Luego se diseñó el prototipo de App para sistema operativo Android.

Resultados: se generó una propuesta de formato de reporte de eventos adversos incluyendo aspectos relacionados con el notificador, el producto sospechoso (suplementos dietarios y nutriciones artificiales) y datos relacionados con el evento adverso. Con esta información se diseñó el prototipo de App para sistema operativo Android.

Conclusión: se generó un prototipo de aplicación para Android, que se ajusta a las necesidades de generación de reportes de eventos adversos de suplementos dietarios y nutriciones artificiales, y que se encuentra acorde con el proceso de nutrivigilancia de la agencia ANSES de Francia y la FDA de Estados Unidos.

Palabras clave: nutrivigilancia, eventos adversos, suplementos dietarios, nutriciones artificiales, prototipo de aplicación.

\section{CARACTERIZACIÓN ANTROPOMÉTRICA DE ESTUDIANTES UNIVERSITARIOS POR EL MÉTODO DE FRACCIONAMIENTO DE LA MASA CORPORAL EN 5 COMPONENTES}

Maximiliano Kammerer López

Objetivo: caracterizar antropométricamente los estudiantes del pregrado de nutrición y dietética de la universidad CES por medio del método antropométrico bicompartimental y el método antropométrico de fraccionamiento de la masa corporal por cinco componentes.

Metodología: estudio descriptivo de corte transversal, en el que se incluyeron a todos los estudiantes del programa de Nutrición y Dietética de la Universidad CES. La fuente de información fue primaria, a partir de la aplicación de un instrumento que permitió realizar la valoración nutricional de los estudiantes a través de mediciones antropométricas mediante los parámetros establecidos por la Sociedad Internacional para el desarrollo de la Kineantropometría. El análisis de la información se realizó a través de la estadística descriptiva básica mediante el análisis cuantitativo de las variables.
Resultados: el $86,6 \%$ de la población de estudio estuvo compuesta por mujeres, el $52 \%$ de ellas presentó un nivel de actividad física liviano, mientras que el $48 \%$ de los hombres presentó un nivel de actividad física intenso. Los valores de porcentaje de grasa para el modelo de dos componentes, difiere entre hombres y mujeres. Comparando el método de 5 componentes con el modelo bicompartimental se observa que el porcentaje de masa adiposa es mayor en ambos sexos que el porcentaje de grasa.

Conclusión: los resultados obtenidos permitieron corroborar que la metodología de fraccionamiento de la masa corporal por cinco componentes es mucho más específica ya que evalúa tejido muscular, óseo y adiposo que están directamente relacionados con el estado de salud y desempeño físico de cualquier individuo. 


\section{ASOCIACIÓN DE LA ACTIVIDAD FÍSICA Y LA ALIMENTACIÓN CON EL RENDIMIENTO ACADÉMICO EN ESTUDIANTES UNIVERSITARIOS: UN ESTUDIO DE COHORTE}

Gustavo Alfonso Díaz Muñoz

Introducción: la asociación de la actividad física (AF) y la alimentación con el rendimiento académico ha sido poco estudiada en universitarios.

Objetivos: cuantificar la asociación entre el rendimiento académico con el nivel de actividad física y el consumo diario de grupos de alimentos en estudiantes universitarios de I-IV semestre durante el segundo semestre de 2017.

Métodos: estudio de cohorte prospectiva. Se convocó a toda la población para participar en el estudio. Se midió la alimentación mediante un cuestionario cualitativo de frecuencia de consumo de alimentos y la actividad física con el IPAQ-SF. El desenlace fue el rendimiento académico medido con la calificación al final del semestre. Se desarrollaron modelos lineales y logísticos multivariados.

Resultados: se siguieron 1274 estudiantes, 33\% hombres (IC 95\%: 30\%35\%) y mediana de edad 19 años (P2518-P7520; IC 95\%: 19,05-19,33). Los

Grupo de investigación en nutrición, genética y metabolismo. Facultad de Medicina. Universidad El Bosque

diazgustavo@unbosque.edu.co niveles de AF fueron: bajo 45,2\% (IC 95\%: 42,5\%-47,9\%), medio 20,5\% (IC 95\%: $18,3 \%-22,7 \%$ ) y alto $34,3 \%$ (IC 95\%: 31,7\%-36,9\%); los dos grupos de alimentos con mayor consumo diario fueron: lácteos 47,6\% (IC 95\%: 44,8\%-50,3\%) y cereales $45,1 \%$ (IC 95\%: 42,3\%-47,8\%). Las variables asociadas a mayor rendimiento académico fueron: consumo habitual del desayuno $(\beta: 0,11$ IC 95\%:0,04-0,18; OR:2,4 IC 95\%:1,3-4,2), consumo habitual de media mañana ( $\beta: 0,06$ IC 95\%:0,01-0,11; OR:1,6 IC 95\%:1,1-2,5), consumo diario de frutas ( $\beta: 0,08$ IC 95\%:0,03-0,13; OR:1,6 IC 95\%:1,1-2,5), AF baja ( $\beta: 0,12$ IC 95\%:0,070,18; OR:2,3 IC 95\%:1,4-3,8) y AF media ( $\beta: 0,14$ IC 95\%:0,08-0,21; OR:2,6 IC $95 \% 1,5-4,7)$. No se encontraron interacciones y el nivel alto de AF presentó colinealidad con los demás niveles de AF.

Conclusiones: en la población estudiada se encontró que el mayor rendimiento académico está asociado con el consumo de desayuno, media mañana, frutas y niveles bajos o intermedios de actividad física.

Palabras clave: Alimentación saludable, ejercicio, estudiantes, universidades, rendimiento académico.

\section{PREVALENCIA DE SARCOPENIA SEGÚN EL ALGORITMO EWGSOP2 EN ADULTOS MAYORES DE UN CENTRO GERONTOLÓGICO DE LA CIUDAD DE GUAYAQUIL}

Ludwig R. Álvarez Córdova, Diana Fonseca Pérez, Jestin Quiroz Brunes, Doménica Salcedo Martinez

Objetivo: determinar la prevalencia de sarcopenia en adultos mayores según el algoritmo de la EWGSOP2 que asisten a un centro gerontológico de la ciudad de Guayaquil.

Métodos: el estudio fue de tipo descriptivo, observacional transversal de diagnóstico y estadificación de la sarcopenia en el que participaron 65 adultos mayores de 65 años que acuden a un centro gerontológico de la ciudad de Guayaquil. Se utilizó los criterios del EWGSOP2 valorando la fuerza prensil de mano con un dinamómetro marca Jamar, la masa múscular apendicular utililzando una bioimpedancia eléctrica marca seca mBCA 525 y la funcionabilidad muscular utilizando el Short Battery Test.

Resultados: el 94\% de los AM fueron mujeres y el resto hombres, la edad promedio fue $76,61 \pm 6,25$ años, el peso fue $63,75 \pm 10,64 \mathrm{~kg}$ y la talla 1,52 \pm 0,07 m. La capacidad funcional se encontró disminuida en $90,77 \%$ de los AM. Se diagnosticó probable sarcopenia en todos los AM varones, y en el 51\% de las mujeres; la sarcopenia establecida y sarcopenia severa se diagnosticó en $10 \%$ y $20 \%$ respectivamente de las mujeres. Se indentificó probable sarcopenia en $54 \%$, sarcopenia establecida en $9 \%$ y sarcopenia severa en $18 \%$ de los AM.

Conclusiones: la probabilidad de presentar sarcopenia fue la condición más frecuente de la población total con más de la mitad de los AM, la prevalencia de sarcopenia estableciada y severa no supera al $33 \%$ reportada en centros gerontológicos.

Palabras clave: sarcopenia, adulto mayor.

Universidad Católica de Santiago de Guayaquil, Ecuador.

\section{EFECTO DE LA QUIMIOTERAPIA SOBRE LA PERCEPCIÓN DEL SENTIDO DEL GUSTO, PESO CORPORAL Y CALIDAD DE VIDA EN PACIENTES CON CÁNCER DE MAMA}

Vanessa Fuchs Tarlovsky, Mónica Patricia Bejarano Rosales, Karolina Alvarez Altamirano, Sofía Payró Katthain

Introducción: los trastornos relacionados con la percepción de los sabores básicos se clasifican en aguesia, hipoguesia y disguesia. Aproximadamente el $69 \%$ de las pacientes con cáncer de mama ( $\mathrm{Ca} \mathrm{Ma)} \mathrm{durante} \mathrm{el} \mathrm{tratamiento} \mathrm{de}$ quimioterapia (Qt) padecen alguna alteración del sentido del gusto.

Objetivo: evaluar los cambios en la percepción del sentido del gusto y su relación con los cambios en el peso corporal y calidad de vida (QoL) en pacientes con CaMa en Qt neoadyuvante.

Métodos: estudio de cohorte prospectivo, observacional, longitudinal y analítico. Pacientes con CaMa EC IIA IIB y III, que inicien Qt neoadyuvante en el Hospital General de México "Dr. Eduardo Liceaga"

Hospital General de México, México.
Resultados: se estudiaron 35 mujeres de $48.8 \pm 7.3$ años. Los cambios en la percepción del sentido del gusto con base al tipo de sabor (dulce, salado, ácido y amargo) y concentración de cada sabor (1\%, $2.5 \%$ y $5 \%$ ) no presentaron cambios significativos; así como su relación con las variaciones en el peso corporal o la dosis de quimioterapia ( $p>0.05 \%$ ). De la QoL la función social, la fatiga y nauseas mostraron una diferencia significativa al finalizar el tratamiento $(p<0.05)$.

Conclusiones: se observó que, a pesar de no alcanzar significancia estadística, después del ciclo de Qt a mayor concentración de los sabores dulce y salado aumenta la sensación de disgeusia. El sabor que presentó mayor hipogeusia fue el ácido y la concentración al 5\% del sabor amargo disminuyó la disgeusia el $8.5 \%$ en relación con el inicio del estudio.

Palabras clave: Alteración sentido gusto, cáncer mama, quimioterapia. 


\section{COMPOSICIÓN CORPORAL Y ESTADO NUTRICIONAL DE PACIENTES CON ENFERMEDAD PULMONAR OBSTRUCTIVA CRÓNICA DE MANIZALES}

\section{Clara Helena González Correa}

Introducción: la Enfermedad Pulmonar Obstructiva Crónica (EPOC), puede causar efectos sistémicos significativos como pérdida de la masa muscular esquelética, lo que conlleva a limitaciones en la realización de ejercicio y calidad de vida. Se ha sugerido incluir en la valoración de estos pacientes, el análisis de la composición corporal y el estado nutricional, con el fin de realizar una rehabilitación integral.

El objetivo del presente estudio fue evaluar el estado nutricional y establecer la composición corporal de los pacientes con EPOC de la ciudad de Manizales. Se utilizó el índice de masa libre de grasa (IMLG), el porcentaje de grasa y el IMC. La estimación del IMLG se analizó mediante análisis de bioim-

Universidad de Caldas, Colombia. pedancia eléctrica y se definió bajo, cuando era menor de $15 \mathrm{~kg} / \mathrm{m}^{2}$ en mujeres y menor de $16 \mathrm{Kg} / \mathrm{m}^{2}$ en hombres. El IMC se evaluó y clasificó de acuerdo a la OMS y el porcentaje de grasa a partir de pliegues cutáneos mediante la fórmula de Durnin y Womersley (1974).

Resultados: 55 pacientes cumplieron los criterios de inclusión. La mayoría presentaban IMC normal 49.1\% (n: 27), el 43,6\% (n: 24) tenían sobrepeso u obesidad y solo el $7,3 \%(n: 4)$ presentaba bajo peso y solo el $37 \%$ de pacientes presentaron un IMLG bajo.

Conclusión: por los hallazgos descritos anteriormente es que es importante evaluar la composición corporal y el estado nutricional de estos pacientes, con el fin de contribuir al tratamiento integral por medio de intervenciones que mejoren la calidad de vida y estado de salud del paciente.

Palabras claves: EPOC, composición corporal, estado nutricional.

\section{DESCRIPCIÓN DE LA PREVALENCIA DE DESNUTRICIÓN CRÓNICA EN RELACIÓN CON EL CONSUMO DE GRUPOS DE ALIMENTOS, EN NIÑOS VÍCTIMAS DEL CONFLICTO ARMADO EN COLOMBIA, 2018}

María del Mar Arango Posada, María Adelaida Atuesta Londoño, Uriel Palacios Barahona

Introducción: los niños que han sido víctimas del conflicto armado, pueden tener mayores deficiencias nutricionales. El bajo consumo este tipo de alimentos, es un factor que se asocia al desarrollo de desnutrición crónica, factor predictivo de peores resultados cognitivos.

Objetivo: determinar la prevalencia de desnutrición crónica en niños víctimas del conflicto armado.

Metodología: este estudio evaluó 125 niños entre los 1 y 5 años. Se tomaron datos socio demográficos y antropométricos (peso y talla). La ingesta se cuantificó con una encuesta de frecuencia de alimentos, para analizar la relación entre algunas variables y el estado nutricional, se utilizó la prueba chi cuadrado.

Resultados: la muestra consistió en 125 niños víctimas del conflicto armado de 1 a 5 años residentes de la ciudad de Medellín, Colombia. El 100
\% de la población pertenecía a estrato socioeconómico bajo. El 32,8\% de los niños presentaron desnutrición crónica. El 52,8\%, 30,8\%, 20,0\% y 13,2\% de los niños consumen menos de una porción de alimentos proteicos, verduras, frutas, y lácteos o sus derivados, respectivamente al día. Se encontró relación entre la presencia de desnutrición crónica y el no consumo de una dieta con una mínima cantidad de alimentos proteicos, vegetales y frutas y lácteos o sus derivados $(p=0,001)$.

Conclusiones: en este estudio se evidenció una prevalencia tres veces mayor de desnutrición crónica con respecto a la población de niños colombiana5, asociada al consumo deficiente de alimentos proteicos, frutas, verduras y lácteos.

Palabras clave: desnutrición crónica, víctimas, niños, retraso del crecimiento.

Universidad CES, Medellin, Colombia

\section{CARACTERIZACIÓN DE PACIENTES CRÍTICOS QUE RECIBIERON SOPORTE NUTRICIONAL EN UN HOSPITAL DE III NIVEL}

Sandra Milena Rojas, Rolando Medina, Juan Sanjuan, Justo German Olaya, Wilmer Botache

Introducción: las necesidades nutricionales en los enfermos críticos no se comprenden bien y varían según la fase de la enfermedad crítica. Aunque los resultados no se han estudiado adecuadamente en los ensayos aleatorios, el objetivo principal del apoyo nutricional es alterar el curso y el resultado de la enfermedad crítica. La fase de recuperación que comienza a medida que se resuelve la enfermedad crítica se caracteriza por un anabolismo que excede el catabolismo.

Objetivo: caracterización de los pacientes críticos que recibieron soporte nutricional.

Método: estudio descriptivo, observacional, retrospectivo. Datos analizados en SPSS versión 21.

Resultados: se incluyeron 542 pacientes, el 57\% correspondió a el sexo masculino, edad media de 56 años, rango entra 14 y 97 años, peso promedio

Universidad Surcolombiana, Neiva, Colombia. Departamento de Cirugía general,

Universidad Surcolombiana, Neiva, Colombia.
65 kg, el 79,2 \% recibieron nutrición enteral y el 3,1\% nutrición mixta, vía de administración: sonda nasogástrica $57,7 \%$, gastrostomía $6,8 \%$ y sonda orogástrica $16,4 \%$, el $14,2 \%$ recibieron formulas poligoméricas, $5,1 \%$ oligoméricas y 80,7\% especiales, días de alimentación mínimo 2 máximo 187 días, glicemia máxima 502 media de 134, creatinina máxima de 23,3, media de 1,3, se realizó prueba de chi cuadrado encontrando una relación estadísticamente significativa entre vía de alimentación y días de alimentación con una p de 0,000, uso de inotrópico y mortalidad p 0,000.

Conclusiones: el soporte nutricional continua siendo una herramienta fundamental para pacientes críticos ayudando a la modulación de su respuesta inflamatoria, así mismo la adecuada elección de soporte nutricional hace la diferencia a largo plazo.

Palabras clave: soporte nutricional, paciente crítico. 


\title{
CAMBIOS EN ESTILO DE VIDA EN PACIENTES CON RECURRENCIA DE CÁNCER
}

Laura Castañeda Gaviria', Gloria Cecilia Deossa R², Luis Fernando Restrepo B³

Introducción: por el aumento de la incidencia de cáncer a nivel mundial y de su supervivencia, se pretende describir los cambios en el estilo de vida tras un primer diagnóstico de cáncer, que realizan quienes presentan recurrencia y reciben tratamientos para este en centros especializados de la ciudad de Medellín.

Métodos: La información se recopiló mediante una encuesta que indaga los datos sociodemográficos, el tipo de cáncer (primario y secundario), los antecedentes personales y familiares de salud y los cambios en actividad física, horas de sueño, consumo de cigarrillo y de licor.

Resultados: se encontró como resultados gracias a la participación de 195 personas, que el $67 \%$ eran sexo femenino, la mayoría pertenecían al régimen contributivo de afiliación a la salud y la mitad pertenecían al estrato socioeco- nómico medio. Los tratamientos más frecuentes para el cáncer fueron la quimioterapia y la cirugía. El antecedente personal más común para ambos sexos fue la hipertensión, seguido de la diabetes, la obesidad y los problemas cardiovasculares. El cáncer de mama y colon fueron los más prevalentes en mujeres; mientras que en los hombres fueron colon y próstata; el cáncer de pulmón fue significativamente mayor en hombres, mientras que el de piel no se presentó en este sexo.

Conclusiones: tras el segundo diagnóstico, aumentó la práctica de actividad física, disminuyó el consumo de cigarrillo y las horas de sueño diario. Se deben hacer cambios permanentes en estilos de vida y preferiblemente de forma preventiva para evitar la incidencia de cáncer y más aun la recurrencia.

Palabras clave: cáncer, recidiva, sobrevivientes, estilo de vida

Coomulsap, Medellín

Escuela de Nutrición y Dietética, Universidad de Antioquia

Facultad de Ciencias Agrarias, Universidad de Antioquia, Medellín, Colombia.

\section{FLAVONOIDES CON ACTIVIDAD ESTROGÉNICA EN UN COMPLEMENTO NUTRICIONAL INFANTIL, A BASE DE AISLADO DE PROTEÍNA DE SOYA}

\author{
Sandra Milena Duitama Carreño', Wilson Alfonso Mejía Naranjo', Leopold Ilag Luna², Javier Zurita²
}

Introducción: la soya y sus derivados, además de tener una proteína de alta calidad, contienen isoflavonoides como las isoflavonas, compuestos con actividad estrogénica que han generado controversia por estar asociados con efectos sobre el comienzo de la pubertad. El contenido de isoflavonas en productos comerciales a base de soya no se presenta en las etiquetas, posiblemente debido al hecho que, las casas comerciales no cuantifican estos compuestos y por ende desconocen la cantidad de los mismos.

Objetivo: evaluar el contenido de isoflavonas gliconadas, agliconadas y totales en un complemento nutricional para niños, a base de aislado de proteína de soya.

Métodos: para ello, se analizaron 3 muestras del complemento nutricional. Se identificaron las isoflavonas presentes en las muestras por cromatografía

Pontificia Universidad Javeriana, Bogotá, Colombia.

Universidad de Estocolmo, Estocolomo, Suecia. líquida acoplada a espectrometría de masas tándem y se cuantificaron por el método de adición de estándar. Las isoflavonas identificadas en el complemento nutricional fueron, las gliconas genistina, daidzina y glicitina y las agliconas genisteina y daidzeina.

Resultados: el contenido de isoflavonas totales encontrado fue de 78 a 98 $\mu \mathrm{g} / \mathrm{g}$ y el consumo estimado al día de 3,5 a 4,4 mg/día, este valor se encontró por debajo del recomendado para niños en edad prepuberal, de acuerdo con valores de referencia reportados en la literatura, extrapolados de los valores recomendados para población adulta; ya que hasta el momento, no se han establecido claramente valores de referencia para este grupo etario.

Palabras clave: isoflavonas, complemento nutricional, aislado de proteína de soya.

\section{COMPOSICIÓN CORPORAL Y ESTADO NUTRICIONAL DE PACIENTES CON ENFERMEDAD PULMONAR OBSTRUCTIVA CRÓNICA DE MANIZALES}

Clara Helena González Correa

Introducción: la Enfermedad Pulmonar Obstructiva Crónica (EPOC), puede causar efectos sistémicos significativos como pérdida de la masa muscular esquelética, lo que conlleva a limitaciones en la realización de ejercicio y calidad de vida. Se ha sugerido incluir en la valoración de estos pacientes, el análisis de la composición corporal y el estado nutricional, con el fin de realizar una rehabilitación integral.

Objetivo y método: el presente estudio utilizó el índice de masa libre de grasa (IMLG), el porcentaje de grasa y el IMC para establecer la composición corporal y el estado nutricional de los pacientes con EPOC de la ciudad de Manizales. La estimación del IMLG se analizó mediante análisis de bioimpedancia eléctrica y se definió bajo, cuando era menor de $15 \mathrm{~kg} / \mathrm{m}^{2}$ en mujeres y menor de $16 \mathrm{Kg} / \mathrm{m}^{2}$ en hombres. El IMC se evaluó y clasificó de acuerdo a la OMS y el porcentaje de grasa a partir de pliegues cutáneos mediante la fórmula de Durnin y Womersley (1974). 55 pacientes cumplieron los criterios de inclusión. La mayoría presentaban IMC normal 49.1\% (n: 27), el 43,6\% (n: 24) tenían sobrepeso $u$ obesidad y solo el $7,3 \%(n: 4)$ presentaba bajo peso y solo el $37 \%$ de pacientes presentaron un IMLG bajo.

Conclusiones: por los hallazgos descritos anteriormente es que es importante evaluar la composición corporal y el estado nutricional de estos pacientes, con el fin de contribuir al tratamiento integral por medio de intervenciones que mejoren la calidad de vida y estado de salud del paciente.

Palabras claves: EPOC, composición corporal, estado nutricional, Manizales. 
- Total de trabajos inscritos al concurso: 77

- Protocolos de investigación: 20

- Trabajos de grado: 14

- Trabajos libres: 43

- Total de posters: 11

Se publican los resúmenes que fueron enviados en el tiempo estipulado. La Tabla 1 muestra los trabajos ganadores.

Tabla 1. Ganadores del premio José Félix Patiño Restrepo

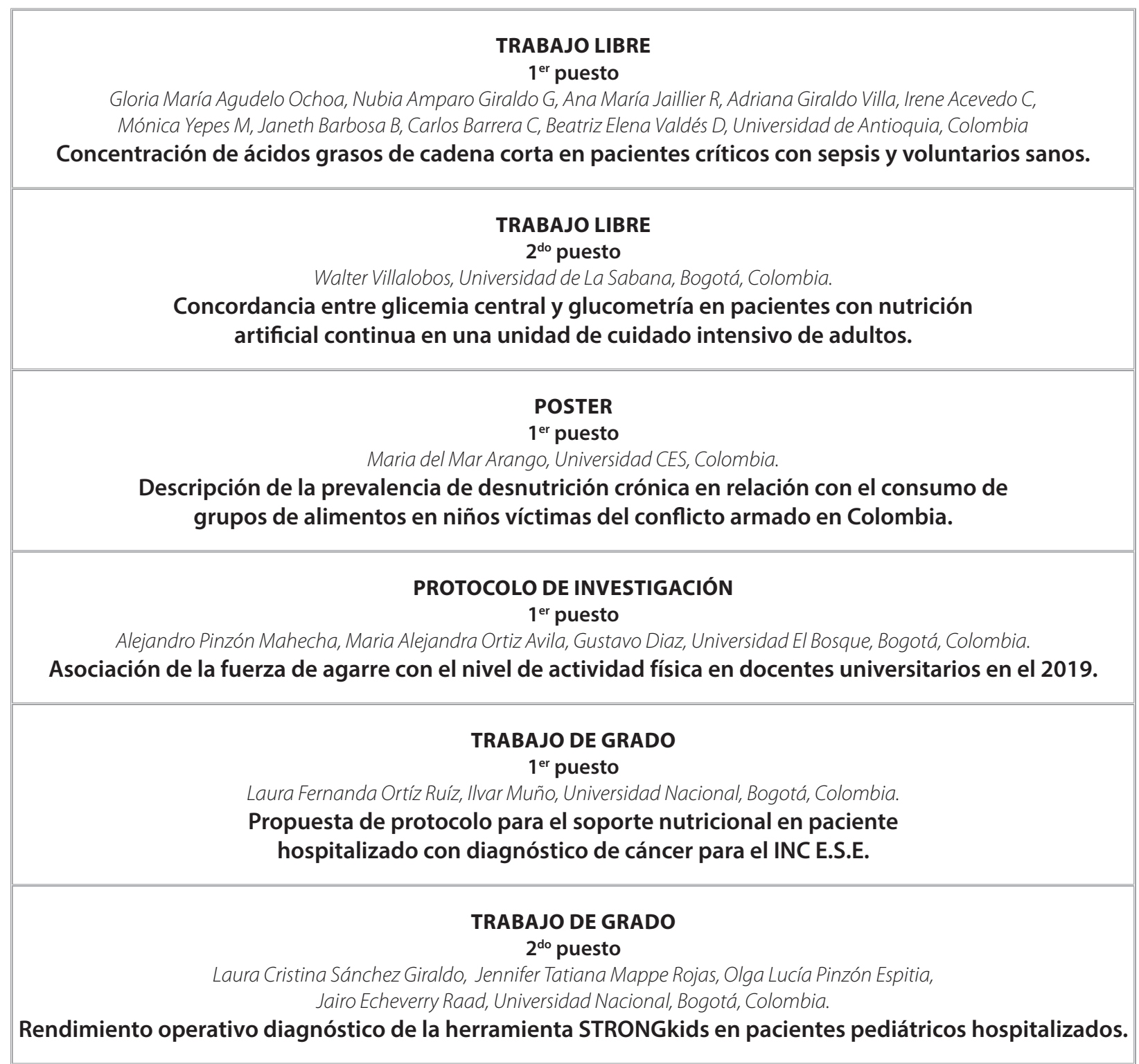

